\title{
Finding Your Way through the Rijksmuseum with an Adaptive Mobile Museum Guide
}

\author{
Willem Robert van Hage ${ }^{1}$, Natalia Stash ${ }^{2}$, Yiwen Wang ${ }^{2}$, and Lora Aroyo ${ }^{1}$ \\ 1 VU University Amsterdam \\ wrvhage@few.vu.nl, l.m.aroyo@cs.vu.nl \\ 2 Eindhoven University of Technology \\ n.v.stash@tue.nl, y.wang@tue.nl
}

\begin{abstract}
This paper describes a real-time routing system that implements a mobile museum tour guide for providing personalized tours tailored to the user position inside the museum and interests. The core of this tour guide originates from the CHIP (Cultural Heritage Information Personalization) Web-based tools set for personalized access to the Rijksmuseum Amsterdam collection. In a number of previous papers we presented these tools for interactive discovery of user's interests, semantic recommendations of artworks and art-related topics, and the (semi-)automatic generation of personalized museum tours. Typically, a museum visitor could wander around the museum and get attracted by artworks outside of the current tour he is following. To support a dynamic adaptation of the tour to the current user position and changing interests, we have extended the existing CHIP mobile tour guide with a routing mechanism based on the SWI-Prolog Space package. The package uses (1) the CHIP user profile containing user's preferences and current location; (2) the semantically enriched Rijksmuseum collection and (3) the coordinates of the artworks and rooms in the museum. This is a joint work between the Dutch nationally funded CHIP1 and Poseidor 2 projects and the prototype demonstrator can be found at http://www.chip-project.org/spacechip.
\end{abstract}

Keywords: Interactive museum tours, mobile museum guide, semantic web, recommender systems, user modeling.

\section{Introduction}

Cultural heritage and museum collections provide a wide variety of objects, which could be of interest to different visitors. To meet the diversity of preferences and backgrounds of visitors museum curators offer tours on different topics. However, these topics usually are selected based on the highlights of the collection and the resulting tours include a fixed and predefined sequence of artworks to

\begin{tabular}{ll}
\hline http://www.chip-project.org \\
2 http://www.esi.nl/poseidon
\end{tabular}

L. Aroyo et al. (Eds.): ESWC 2010, Part I, LNCS 6088, pp. 46-59, 2010.

(C) Springer-Verlag Berlin Heidelberg 2010 
view. An audio tour provides more freedom in determining your own sequence of artworks while visiting a museum. However, the set of artworks to choose from is still a predefined one and is the same for all visitors. Currently, museums turn to multimedia guides in order to bridge the gap between the visitor's interests and the static museum tours. Personalization is one way to provide dynamics related to visitor's interests, which subsequently could enhance visitor's experiences [5]. An adaptive mobile museum guide acts as a museum expert and provides the user with information adapted to the current situation [2]. For example, the MIT Media Lab ${ }^{3}$ audio and visual narration adapts to the user's interest acquired from the physical path in the museum and length of the user stops. The mobile museum guides developed within Hippie [3] and PEACH [4] projects provide content adaptation based on technical restrictions of specific presentation devices as well as visitor's preferences and knowledge. The difference between two projects is that Hippie museum guide uses stationary and mobile devices in a sequential way (e.g., a user prepares his museum visit on the personal computer at home and then uses the mobile device while actually visiting the museum), the PEACH museum guide combines both mobile and stationary devices in parallel. The mobile museum guide built within Sotto Voce [1] project takes into account the special needs of groups visiting a museum and facilitates social interaction between group members. AgentSalon [6] system users are provided with mobile devices and are monitored while exploring the museum. The system can infer an overlap between users' interests and experiences and fosters communication between the users with stationary devices. ARCHIE [10] provides a socially-aware handheld guide that stimulates interaction between group members. They can communicate with each other either directly (by voice) or indirectly (by collaborative games) by means of their mobile guides. By using a personal profile it allows to adapt the interface and tailor the information to the needs and interests of each individual user. The user profile evolves slowly by observing how the user interacts with the digital content, e.g. asking for more, or bookmarking it, may indicate interest while stopping an explanation prematurely may indicate a lack of it. The Kubadji mobile tour guide 4 aims at deriving visitor's interests from implicit behavior (e.g. artworks viewing times), recommendation of items of interest and personalization of the content delivered for these items via the handheld device. Besides it uses collaborative filtering approach for predicting visitor's viewing times of unseen exhibits from his viewing times at visited exhibits. The context-aware museum tour guide presented in [11] is used to give directions to the visitor and is adjusted as the tour progresses dropping one or more exhibits if the visitor falls behind the tour or suggesting additional exhibits or taking a break at a nearby restaurant if the visitor has extra time. The environment also supports peer-to-peer interactions between visitors, allowing them to find each other, share ratings and comments about exhibits. A number of museums, e.g. Tate Modern, Science Museum Boston, are already exploring the potential of personalized museum guides, currently available on their websites.

\footnotetext{
3 http://www.media.mit.edu/

4 http://www.kubadji.org/
} 
A major bottleneck in realization of this personalization is how to collect the necessary information about the user's (constantly evolving) interests [5] without intruding on the visitor too much. Typically, for large scale online access personalization can be achieved through usage of stereotypes (e.g. students, novices, art experts, children) or through deducing a user profile from observation of their online browsing and searching (or in museum) viewing behavior. In this way, personalized virtual tours are ways for visitors to construct their own narratives. In addition, the indoor localization of people and objects plays a critical role in order to implement and deploy successfully such a system. Two tasks are considered in this context [2]:

- Detecting user's location inside the museum requires a positioning system that considers the boundaries and constraints (i.e. the walls, doors, stairs) of the physical indoor space. Methods using different hardware solutions have been proposed to increase the accuracy of the indoor user position.

- Assessing user's context in terms of artworks in her neighborhood, which artworks have been already seen by the users, how much time has the user already spent in the museum and additional temporal constraints (e.g. how much time is available), what are visitor's general interests in art, and potentially also their physiological and the emotional state [2].

Having the limited resources of mobile guides in mind, most of representation and processing of relevant knowledge needs to be carried out remotely in the infrastructure. To reduce complexity and to ensure reusability of the knowledge representations and inference mechanisms a flexible web-based approach is required that allows different types of systems to exchange and augment information on users and particular situations 2. In the following sections we discuss briefly the CHIP project, the routing mechanism of SWI-Prolog Space package and pay a special attention to the SPACE-CHIP demonstrator.

\section{CHIP Demonstrator}

The CHIP (Cultural Heritage Information Presentation) project is a crossdisciplinary research project, combining aspects from cultural heritage and information technologies. The team has been working at the Rijksmuseum Amsterdam since early 2005, as part of the Dutch CATCH (Continuous Access to Cultural Heritage) program on techniques to provide a personalized access to the museum collection both online and inside the museum, therefore also allowing to link visitor's experience in both environments. We have used explicit semantics in order to enrich the museum collection and in this way to be able to discover relationships between the objects in the collection, and use those to generate personalized recommendations and tours for each user. The main goal of the project so far, was to explore how explicit semantics can improve the users' satisfaction with respect to the recommendation of artworks and related art concepts. All

\footnotetext{
${ }^{5}$ Virtual Museum (of Canada), http://www.museevirtuel-virtualmuseum.ca/
} 
the CHIP Web-based tool 6 for browsing the online collections, planning personalized museum tours, getting recommendations about interesting artworks to see, and quickly finding their ways in the museum use a common distributed user model, which collects user interaction data and interprets it in terms of user's interests used further for generating recommendations and personalized tours. The Art Recommender helps the user to discover his art interests in the Rijksmuseum collection and store them in a user profile (also visible to the user). The Tour Wizard helps the user to generate personalized tours through the museum based on his user model and visualizes them on the museum map and on a historical timeline. By default, two tours are generated automatically for each user based on his ratings-Tour of Favorites containing artworks positively rated by the user and Tour of Recommended Artworks containing 20 top recommended artworks. The user can also create tours by searching for concrete artworks. The e-Culture Semantic Search 7 open API helps him find semantically related topics and artworks to include in the personalized tours. For example, if the user searches for "Rembrandt" he gets not only artworks created by Rembrandt himself but also artworks created by Rembrandt's teachers and students, as well as artworks by artists in the same style as Rembrandt. Finally, the Mobile Museum Guide allows users to access their tours created with the Tour Wizard on their mobile devices in the museum. Technical details about the design and implementation of the CHIP Art Recommender, Tour Wizard and Mobile Museum Guide (ver 0.1 and ver 1.0) can be found in 9. However, so far all those tools considered only user preferences are constraints for the adaptation. In order to realize this in the real physical space we need to also consider spatial constraints in generating both recommendations and museum tours. In this paper we propose a method for on-the-fly adaptation of museum tours based on a spatial model of the museum.

The first two versions of the CHIP Mobile Guide 8 used two different hardware settings to allow users to use on their handhelds the museum tours created with the online CHIP Tour Wizard. The Mobile Guide is implemented as a Webbased application and is therefore viewable in any browser. For demonstration purposes we have created also an iPhone simulation of the graphical user interface. The simulation can be used if a user wants to get familiar with the mobile environment before going to the museum. The Mobile Guide automatically detects the screen resolution of the user and based on this information chooses to display the Mobile Guide for a mobile device or the simulation. As the other CHIP tools the Mobile Museum Guide provides an extensive description of each artwork and a set of artworks related to it. Visitors can also search for specific artworks exhibited in the museum and can also give a rating to objects they find interesting or like.

When the user selects a tour to follow the Mobile Guide checks the availability and the positioning of all the artworks in the tour in the museum. It is

\footnotetext{
${ }^{6}$ See http://www.chip-project.org/demo

7 http://e-culture.multimedian.nl/

8 http://www.chip-project.org/demo/mobileguide
} 
possible that the user has created the tour in advance but by the time that he actually managed to visit the museum some artworks could have been removed or replaced. Next the user adds artworks to the tour that qualify according to contextual and user criteria. Similarly the user can remove artworks from the tour. The user can also specify tour constraints, such as the length of the tour in terms of the maximum number of artworks included in a tour or in terms of the maximum time to spend in the museum. Based on these constraints, the tour can adapt dynamically while the user is in the museum. The user can change those adaptation parameters at any point during the usage of the Mobile Guide (e.g. before starting a tour, while following a tour). The user can also choose an option of adapting the tour based on his ratings given to artworks while using a Mobile Guide.

Suppose the user follows a tour of recommended artworks and he has chosen an option of adapting the tour. If he provides a rating to an artwork that he sees CHIP demonstrator updates the user model and as consequence the list of recommended artworks. However, this version of the Mobile Guide does not take spatial constraints as well as information about already seen artworks into account. In section 3 we show how SWI-Prolog Space package allows to solve this problem and in section 4 we present details about the implementation of the third version of the CHIP Mobile guide including those spatial constrains, http://www.chip-project.org/spacechip.

\section{Finding Routes through the Rijksmuseum}

The Art Recommender supplies a list of recommended artworks that are ordered by the estimated likelihood that the user will find them appealing based on manual ratings. Even though the rooms in the Rijksmuseum have themes, such as works about the Dutch republic or works by Rembrandt and his pupils, these themes do not necessarily coincide with the preferences of the user. This means that even a small set of recommended artworks can be distributed over the entire museum. In order to improve the user experience of the museum visit, we reorder the results of the Art Recommender so that there is an efficient way to walk from one to the other. This route minimizes the walking effort, while maximizing the number of top recommendations. Also, it takes into account a optional caps to the walking distance and the number of artworks. This helps the user to decide where to go given limited time. (The feeling of missing something important can cause people to linger too long in the "wrong" rooms and therefore to miss their favorite works.)

An Easy Traveling Salesman Problem. Computing an efficient route through a museum is very similar to the traveling salesman problem. However, for a few reasons, theoretically at least, a significantly easier problem than the general traveling salesman problem, for which the greedy nearest-neighbor search algorithm is considered a sub-optimal solution. First, if you consider the artwork displays, rooms, doors, hallways, and stairs to be nodes in a connectivity graph, 
then this graph is not fully connected, because there are walls and floors in the way. This is illustrated in Fig. 1, Second, the rooms are considered units when the exhibits are created, which means it makes sense to view all works from a single room together. This means it is nearly always a good idea to delay transitions across doors until all displays in a room have been visited. And third, floor transitions take a lot of effort, especially up by stairs, or either way by elevator, because you have to wait for the elevator. For these reasons there are only a few sensible paths through the museum. Locations are grouped per room and then per floor. If you set the transition weight of the edges in the connectivity graph to the experienced distance instead of the actual distance then nearest neighbor search will always send the visitor to works within the same room first before making the transition to another room (or even floor), which is good in the case of the Rijksmuseum, but which is bad in the case the general traveling salesman problem, because it causes local optima.

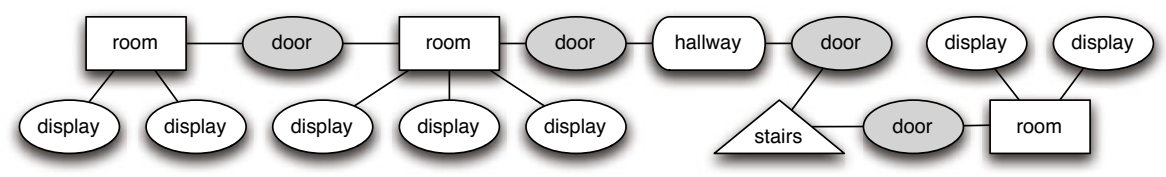

Fig. 1. Example connectivity graph

Implementation of the Nearest Neighbor Router. The SWI-Prolog space package 8] provides nearest neighbor search. However, this nearest neighbor search is unaware of the restrictions posed by the walls and floors. Therefore, we base our routing on a connectivity graph search algorithm that uses intersection queries as opposed to nearest neighbor queries. First we compute a connectivity graph between all the artwork displays, rooms, stairwells, etc. that takes into account where the doors are. Then we compute the weighted shortest path between all the displays. The weight is based on graph distance, the type of the transition (e.g. moving to another floor is more expensive), and on the distance between locations inside a room (e.g. how far displays are from each other or from a door). This shortest distance matrix is used to compute an efficient path along all the recommended artworks. The exact method we use to calculate the routes is as follows:

- Pre-compute artwork distance matrix once

1. define that stairs, hallways, toilets, are rooms

2. define works are at a display in the museum

(a) give the display a $\langle x, y, z\rangle$ coordinate

3. define what it means to be connected

(a) places (displays, doors) space_intersect with same room

(b) places are stated to be connected (stairs to stairs on other floor) by $A$ chip:connectsTo $B$ 
4. assert $A$ chip:connectsTo $B$ for each connected pair $\langle A, B\rangle$

5. make connectivity graph of chip:connectsTo

6. compute weights for each transition

(a) graph distance plus distance within room

(b) door transitions get a higher graph distance than display-display transitions

(c) stairs transitions get an even higher graph distance

7. compute and cache upper triangle matrix of weighted graph shortest path distances between all places

- Apply routing algorithm for each request

1. fetch set of recommended works (given by Art Recommender)

2. fetch current position (given by user interface)

3. fetch remaining time in museum (given by user interface)

4. fetch maximum number of artworks to route (given by user interface)

5. greedy nearest neighbor search in weighted distance graph until list of recommended works is empty:

(a) look up nearest recommended work

(b) remove work from list of candidates

(c) add path from current position to work to recommended route

(d) set current position to location of work

(e) add length of path to total length of recommended route

6. while total path length of recommended route takes longer than remaining time in museum

(a) remove furthest artwork from current position

(b) apply greedy nearest neighbor search again (step 5)

\section{SPACE-CHIP Demonstrator}

\subsection{Usage Scenario}

Imagine the following usage scenario: Our user prepares a visit to the Rijksmuseum. He provides his opinion about a number of Rijksmuseum artworks and topics through the Art Recommender e.g. rates the painting "Woman Reading a Letter" and the artist that made the painting Johannes Vermeer with 4 stars meaning he likes them. These ratings result in the list of recommended artworks that form a Tour of Recommended Artworks that the user can view in the Tour Wizard 9. The user is going to follow this tour inside the museum with the help of the CHIP Mobile Guide. The routed tour is shown in Fig. 3, We use icons

\footnotetext{
${ }^{9}$ For the demonstration purposes we simulate the user's experience with the mobile device by showing the tour map in the Tour Wizard tool. In difference with the original version of the tool in this Tour Wizard we indicate with icons the (imaginary) artworks locations. Semantically enriched data about Rijksmuseum collection only provides information about the room number where a particular artwork is located but does not provide information about the exact artwork location.
} 


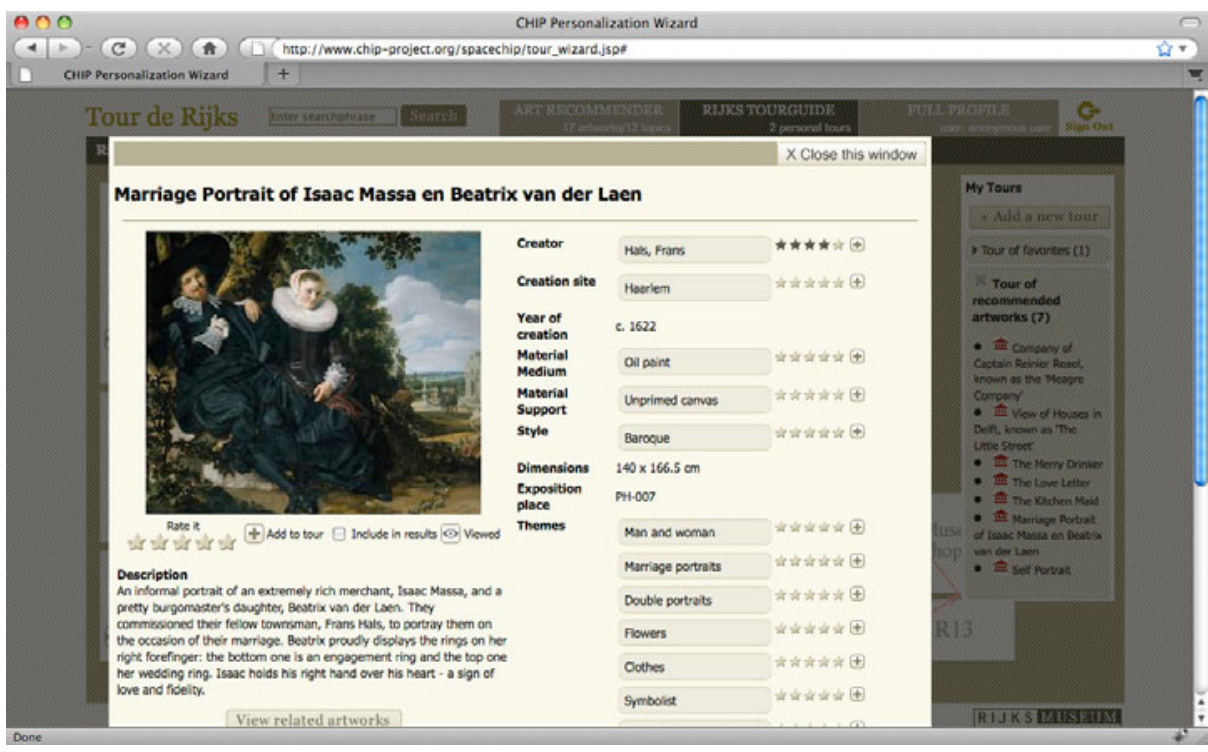

Fig. 2. Artwork description page

in a different color to indicate artworks that are in the tour and connect them with the tour line. The user location is indicated with an icon at the entrance door on the ground floor. During the visit the user views artworks that are in the tour but is also attracted by other artworks outside his tour. In order to give a notification to the system that the user has viewed an artwork he has to click on a corresponding icon on the museum map and in the popup window showing artwork description (see Fig. 2) he has to click on "Viewed" icon. If the user clicks on a "Viewed" icon for an artwork that is in his tour then the tour route remains the same. Otherwise the tour may be re-routed taking into account the user's interest in that artwork. He can also give ratings to any artwork he sees. These actions result in the tour being dynamically adapted taking into account the history of his visit (seen artworks), changing interests and current location. (However if the user wants to follow the initial sequence of recommended artworks and does not want the tour to be adapted he can select a corresponding option in the tour configuration). If the user, for example, likes the works by Frans Hals and Ferdinand Bol he comes across on his way to the recommended Johannes Vermeer works, he can add a rating by selecting the work on the map and submitting a new rating (see Fig. 2). This automatically updates the tour. The updated tour is shown in Fig. 4. For the sake of clarity we have highlighted the works from the original tour with red, the new Frans Hals recommendations with yellow and the new Ferdinand Bol work with blue. 


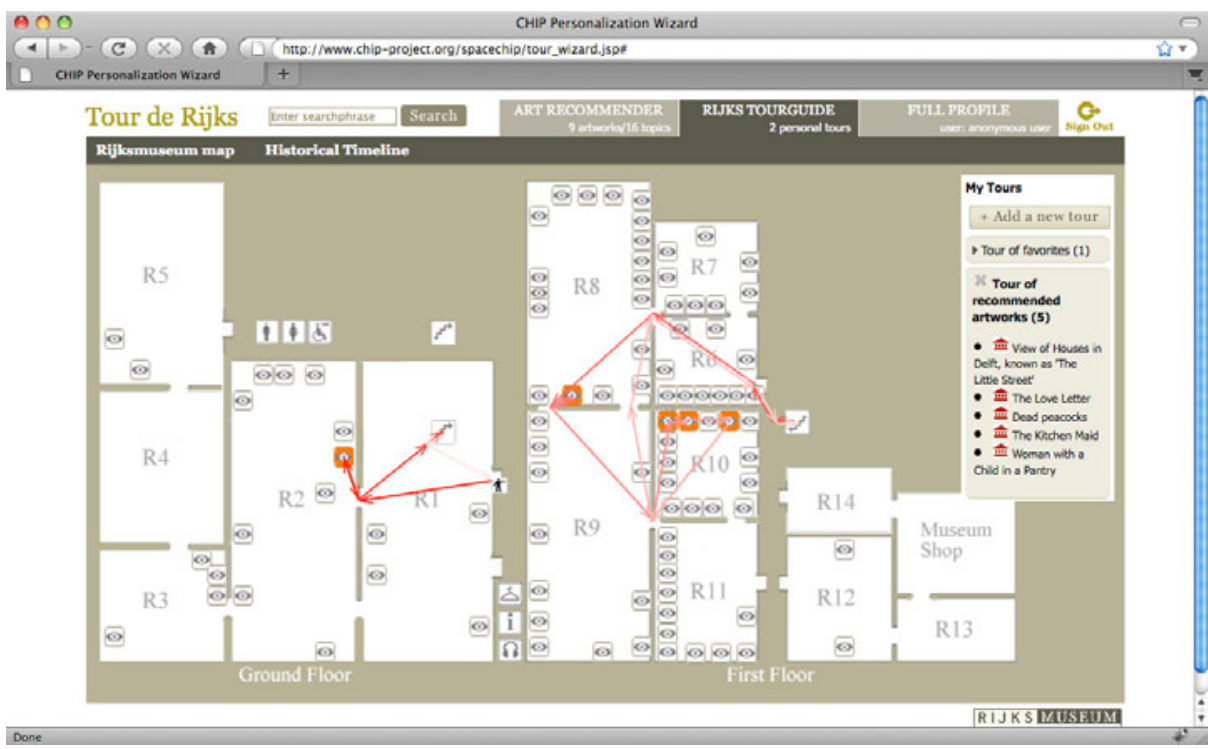

Fig. 3. Initial route of the tour of recommended recommended artworks

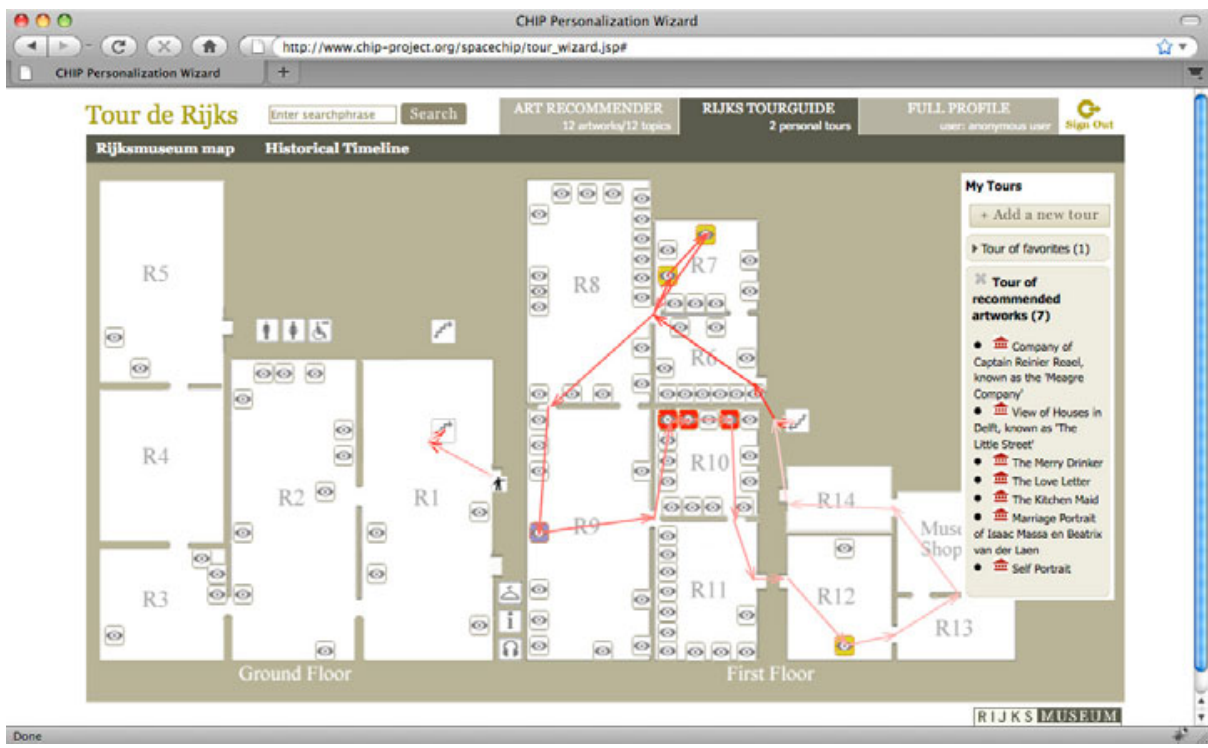

Fig. 4. Re-routed tour of recommended artworks 


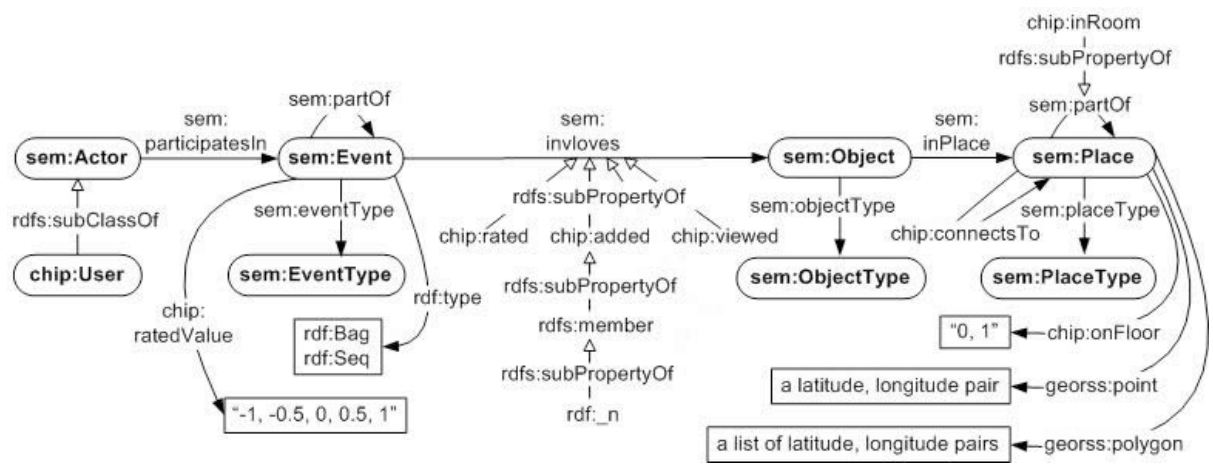

Fig. 5. Mapping CHIP user model (UM) to the simple event model (SEM)

\subsection{Mapping the CHIP User Model to SEM}

In order to provide data exchange between CHIP and SWI-Prolog Space package we mapped the original CHIP user model (UM) 9 specified using RDF/XML to the Simple Event Model (SEM) 10 which is proposed by van Hage et al. 7 . and is just a formalization in RDF using SEM.

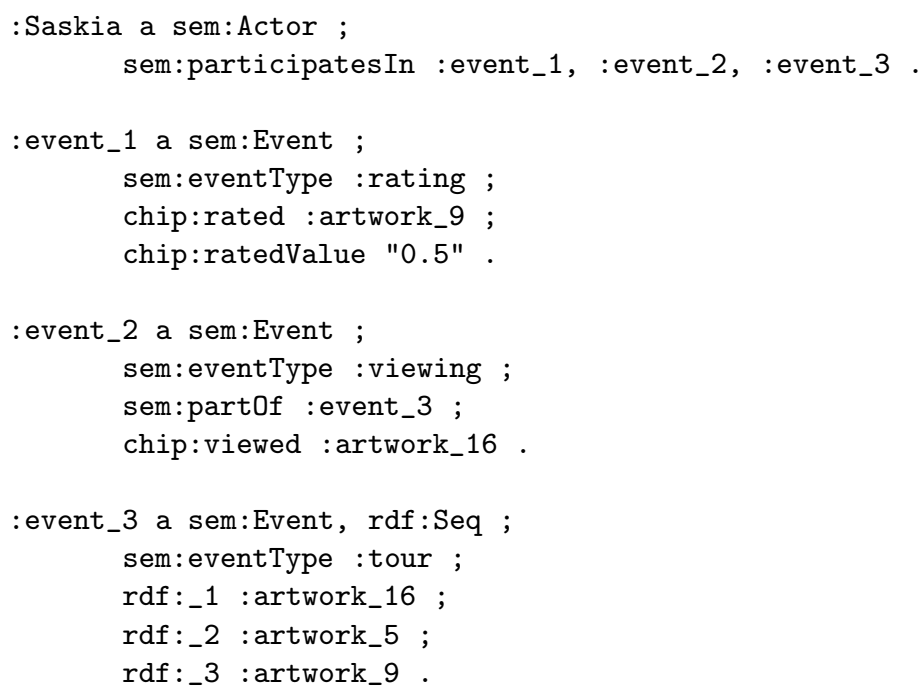

Fig. 6. Code example of the CHIP User Model based on SEM

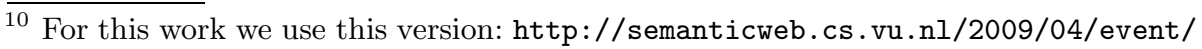
A newer version is available at http://semanticweb.cs.vu.nl/2009/11/sem/ 
As shown in Fig. 5, we defined chip:User as a sub class of the sem:Actor, who participates in the sem:Event. In our case, there are three different types of events: (i) rating, (ii) viewing, and (iii) tour. In a rating event, the user rates a sem:Object with a chip:ratedValue from "-1" to "1". The viewing events are usually part of the tour events, since the user views a sem:Object during the tour. In a tour event, the user adds a sem:Object into a particular tour. All of the objects added in the tour will be ordered in a sequence based on their locations in the museum, which are described using the $\mathrm{rdf}: \mathrm{n}^{11}$ as a sub property of the rdfs:member.

Suppose Saskia is a CHIP user who participated in three events: (i) she rated artwork_9 with a value of "0.5"; (ii) she added three artworks (artwork_5, artwork_9 and artwork_16) in the tour; and (iii) she viewed artwork_16 in this tour. Using the routing algorithm, artworks are ordered in a sequence: artwork_16, artwork_5 and then artwork_9. In Fig. 6 we give the corresponding code that describes these information in the user profile and tour profile.

To indicate the locations of objects in the museum, we use various properties: chip:inRoom, chip:onfloor, georss:point 12 and georss:polygon. There are also many different types of places in our case, such as display (the place type for artworks), room, door, hallway, stair, elevator, restroom, etc. Two places are connected by using the chip: connectsTo property.

\section{Evaluation}

We consider two issues for evaluation: (1) that recommendations are useful for the users and (2) that sequence of recommendations follows an efficient route through the museum in a reasonable time that allows real-time interaction with the system.

With respect to the first issue we performed a study Effectiveness of recommendations, novices versus experts. Our conclusion was that the Art Recommender helps novices to elicit or clarify their art preferences from their implicit or unclear knowledge about the museum collection [9]. Compared to the novices, the experts (mainly museum domian experts) do not seem to benefit from it a lot, although there is a slight increase of 0.23 (the increase for the novices is 1.18) which indicates that the system also helps the experts to elicit their art preferences.

With respect to the second issue, we measured the speed of the router. To determine the speed of the router we measured the CPU time taken on a 2.66 $\mathrm{GHz}$ Intel processor, given enough memory to store the cached distance matrix between the artworks. The result is shown in Fig. 7. Even though the performance curve shows exponential growth in terms of the number of artworks that needs to be routed, the total time needed for the routing stays within reasonable bounds for the number of artworks in a realistic tour through the Rijksmuseum. This performance could be significantly improved by further optimizing the data

\footnotetext{
11 http://www.w3.org/TR/rdf-schema/

12 http://georss.org/simple
} 


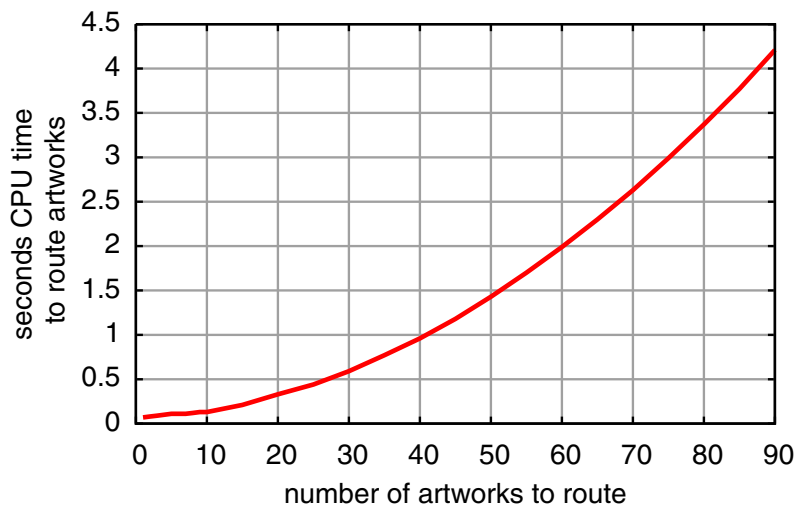

Fig. 7. Time performance of the router for $n$ artworks. The router needs exponential time to derive an efficient route, but stays within a reasonable time for real-time response for the number of artworks that a realistic tour comprises.

structure that stores the distance matrix. At the moment this is a binary tree. An array matrix would provide faster access. Furthermore, we guarantee that the router always favors within-room transitions over between-room transitions, which in turn are always favored over floor transitions. Given the limited connectivity between the rooms and floors this guarantees an efficient path.

\section{Discussion and Future Work}

Existing adaptive mobile museum guides differ in the ways they construct the user model, the ways they provide personalized experience inside the museums, devices that they use. Many projects focus on social communication between the visitors (e.g. friends, group members) while following a tour. Currently, CHIP does not take social aspect into account-neither for generating recommendations, nor for communication inside the museum. This could be one of the improvements to the CHIP demonstrator. The strong points of CHIP however are the distributed user model and the ability to view the CHIP Mobile Guide in any browser. No additional software installation is required while typically museums would provide the visitors with the PDA's running pre-installed software and ask them to provide some personal information to start creating their user models. In the presence of Wifi inside the Rijksmuseum the visitor could use his own device (iPod touch or iPhone) to follow the CHIP Mobile Guide that uses the user model and the tours information stored on the CHIP server.

We consider several directions for future work.

First, implementing the demonstrator for the use in the realistic situation (inside the museum) with the real time data. In the current prototype we simulated the user's experience with the mobile device by showing the tour map in the Tour Wizard tool. To indicate the fact that the user has seen an artwork he has to first 
click on a corresponding icon on the map and then on a "Viewed" icon in the popup window that opens. Time issues while following the tour in this way are not taken into account. The next step in developing the demonstrator would be the implementation of the real-time user localization and re-routing the user by taking into account the time that he spends viewing artworks, moving between artworks, taking stairs, etc.

Second, designing and evaluating the user interface for guiding the user in the realistic situation. At the moment the user is only provided with the museum map that indicates the tour route and the current user location. Based on the map the user has to figure out where to go next. It would be interesting to consider the possibility of guiding the user "locally" by an indication about where to go next from the current point, like turn left/right, etc. It would be also interesting to consider using technologies like Google Goggles 13 to show information about an artwork when the user points with his device on it.

And third, experimenting with various re-routing algorithms. Current algorithm can provide re-routing of a tour or sequencing of a given set of artworks from the tour (generated by CHIP Art Recommender and Tour Wizard tools) based on user's position or closeness to a certain artwork from the tour. In addition to this artworks set, the routing mechanism uses the museum coordinates. It does not take into account the information from the user model. It would be interesting however to consider more complex algorithms that would also take user preferences into account and possibly decide to add more artworks to the tour that might be interesting for the user based on the user closeness to them.

Acknowledgments. The work on the CHIP project is funded by the Dutch Science Foundation CATCH program and is in collaboration with the Rijksmuseum Amsterdam. Part of this work has been carried out as a part of the Poseidon project in collaboration with Thales Nederland, under the responsibilities of the Embedded Systems Institute (ESI). This project is partially supported by the Dutch Ministry of Economic Affairs under the BSIK program.

\section{References}

1. Aoki, P.M., Grinter, R.E., Hurst, A., Szymanski, M.H., Thornton, J.D., Woodruff, A.: Sottovoce: exploring the interplay of conversation and mobile audio spaces. In: SIGCHI Conference on Human factors in computing systems (2002)

2. Kruger, A., Jorg, B., Heckmann, D., Kruppa, M., Wasinger, R.: Adaptive Mobile Guides. In: Brusilovsky, P., Kobsa, A., Nejdl, W. (eds.) Adaptive Web 2007. LNCS, vol. 4321, pp. 521-549. Springer, Heidelberg (2007)

3. Oppermann, R., Specht, M.: A nomadic information system for adaptive exhibition guidance. In: ICHIM99 Conference (1999)

4. Rocchi, C., Stock, O., Zancanaro, M., Kruppa, M., Kruger, A.: The museum visit: generating seamless personalized presentations on multiple devices. In: 9th International Conference on Intelligent User Interfaces (2004)

13 http://www.google.com/mobile/goggles/ 
5. Roes, I., Stash, N., Wang, Y., Aroyo, L.: A personalized walk through the museum: The CHIP interactive tour guide. In: ACM CHI-Student Research Competition (2009)

6. Sumi, Y., Mase, K.: Interface Agents that facilitate knowledge interactions between community members. In: Cognitive Technologies Series, pp. 405-427. Springer, Heidelberg (2004)

7. van Hage, W.R., Malaisé, V., de Vries, G., Schreiber, G., van Someren, M.: Combining ship tra jectories and semantics with the simple event model (sem). In: 1st ACM International Workshop on Events in Multimedia. Sheridan Publishers (2009)

8. van Hage, W.R., Wielemaker, J., Schreiber, G.: The space package: Tight integration of space and semantics. In: 8th International Semantic Web Conference Workshop: TerraCognita (2009)

9. Wang, Y., Stash, N., Aroyo, L., Gorgels, P., Rutledge, L., Schreiber, G.: Recommendations based on semantically enriched museum collections. Journal of Web Semantics 6(4), 43-58 (2008)

10. Luyten, K., Van Loon, H., Teunkens, D., Gabriëls, K., Coninx, K., Manshoven, E.: ARCHIE: Disclosing a Museum by a Socially-aware Mobile Guide. In: 7th International Symposium on Virtual Reality, Archaeology and Cultural Heritage VAST (2006)

11. Chou, S.-C., Hsieh, W.-T., Gandon, F.L., Sadeh, N.M.: Semantic Web Technologies for Context-Aware Museum Tour Guide Applications. In: 19th International Conference on Advanced Information Networking and Applications, pp. 709-714. IEEE Computer Society, Washington (2005) 